\title{
A Retrospective Look At College Football In The Late BCS Era- A Case Study In Sports Analytics, Sports Management, And Sports Economics
}

\author{
Cary A. Caro, Ph.D., Xavier University of Louisiana, USA
}

\begin{abstract}
This case study presents game-play statistics to explore their relationships between winning, conference affiliation, and styles of play. Statistics from games from 2005 to 2011 are compiled and explored using descriptive statistics, ANOVA, and Pearson's Correlations. Bowl Championship Series automatic-qualifiers serve as variables in this study. ANOVA analysis indicates that the style of play from 2005 to 2011 has changed as total offensive production and scoring have increased. This study also finds marked differences in game-play for the various conferences, and presents the statistics that carry the greatest correlation to winning in each of the conferences. This case study is intended for use in sports management and administration courses, where users must make determinations on the differences in play, and their impact on sports administrative decisions.
\end{abstract}

Keywords: Sports Management; Sports Economics; College Football; Bowl Championship Series; Case Study; Athletic Directors; Sports Analytics

\section{THE BOWL CHAMPIONSHIP SERIES (BCS) AND THE BCS CONFERENCES}

The BCS was established in 1998 as a conglomerate of bowl games with the sole purpose of pitting the top two teams in the BCS standings to play for the BCS National Championship (BCS, 2012). The BCS was "an event managed" by the Bowl Subdivision Conferences and the University of Notre Dame. The bowl games included in the series were the Rose, Fiesta, Sugar, and Orange, with an additional game at one of these venues to determine the national champion. Formerly, the BCS was divided into automatic qualifying (AQ) and non-automatic (non-AQ) conferences; however, provisions in 2012 have been made to eliminate those distinctions moving forward.

The revenue impact of the BCS was staggering. In 2011, the BCS distributed \$142.5 million dollars to the different schools in the agreement (SBJ, 2011). These payouts, however, were not equally distributed amongst the conferences, as there are provisions for participation and BCS automatic qualification status. Distribution aside, the BCS opened a revenue stream that allowed many athletic programs to prosper since the series' inception.

Participating in a BCS game created varied opportunities for an athletic program and the university. Aside from the financial payout, the university benefited from the marketing, branding, and publicity that the games and the series created. The conference champions from the AQ conferences (Big East, Big 10, Big 12, SEC, Pac-10, and the ACC) automatically gained a place in one of the bowl games. Conference champions from the non-AQ conferences (Conference USA, Mid-American, Mountain West, Sun Belt, and the Western Athletic) were eligible should they meet the following criteria: be ranked in the top 12 of the final BCS standings or be ranked in the top 16 if that ranking is ahead of the ranking of any of the AQ conference champions (BCS, 2012). Only one of the nonAQ teams could gain an automatic berth to the BCS this way; however, a second team would become eligible should it be ranked in the top 12 of the final BCS standings. 


\section{Winning And Revenue}

The money generated from football plays many roles in athletic departments. Primarily, the money funds non-revenue sports within the department; in addition, the money is used to upgrade facilities, secure coaches to long-term, high dollar salaries, and in some instances, provide money back to the university general fund (Caro \& Benton, 2012). Successful athletic programs also have an impact on alumni donations (Brooker \& Klasorin, 1981; Baade \& Sunderberg, 1996; Rhoads \& Gerking, 2000) and increased giving through athletic funds McCormick \& Tinsley, 1990).

Fulks (2010) reported that college football revenue accounted for nearly $46 \%$ of an athletic department's total revenue. With such a heavy reliance on football revenue, winning is of utmost importance. Padilla and Baumer (1994) indicated that success and television have led to greater profitability for football programs. Caro and Benton (2012) reported a strong, positive relationship between winning, conference membership, and revenue; however, differences in the ability of programs to create football-related revenue have caused great discrepancies between the $\mathrm{AQ}$ and non-AQ conferences and within universities in respective conferences.

The argument can be made that revenue distribution and revenue inequity has challenged the competitive balance on the field. Groza (2010) concluded that teams fare better when they can align themselves with stronger conferences. It was, perhaps, this belief that triggered conference realignment in 2010. The University of Nebraska left the Big 12 in 2010 for the Big Ten, and in the process severed relationships with Oklahoma and Texas, two of their premier, traditional rivals. From 2003 to 2009 the average revenue for teams in the Big 12 was roughly $\$ 27.2$ million; the average revenue for Big Ten teams was nearly $\$ 33.4$ million dollars (Caro \& Benton, 2012). Nebraska (\$36.2 million) ranked fourth in the Big 12, was eclipsed almost two-fold by the University of Texas $(\$ 68.6$ million). While Nebraska called the move to the Big Ten a search for stability and said that they were more "aligned" with the academics, culture, and athletics of the Big Ten (ESPN, 2010), it was hard to ignore the underlying increase in revenue and potential future earnings. Missouri and Texas A\&M made similar departures from the Big 12 in 2011.

Perhaps no conference was hit as hard as the now defunct Big East. West Virginia, Louisville, Syracuse, and Texas Christian (TCU) all departed from the Big East - TCU doing so without ever playing a game in their new conference. Utah departed the Mountain West for the Pac-12. These realignments triggered a race for stability, longevity, and in some instances, survival, as universities sought to establish relationships with conferences that could provide financial security and competitive balance. This recent round of realignment was also a reminder that revenue is of great importance to athletic programs as it provides an opportunity to create a competitive advantage over traditional rivals and the rest of college football. Through this realignment, however, programs never stopped to wonder if their style of play would fit within their new conferences. The question was never asked, "can we fit in and be competitive on the field."

\section{Winning And Recruiting}

The impact that recruiting has on winning cannot be minimized. It stands to reason that teams with a collection of better athletes may have a competitive advantage on the field against a team with inferior talent. While this is not always the case, recruiting plays a pivotal role in on-the-field success. Dumond, Lynch, and Platania (2008) reported a connection between recruiting success and winning: successful teams were recruiting at a higher rate than those who were experiencing a downturn. Caro (2012) supported the findings of Dumond et al. (2008), by reporting a positive relationship between recruiting and revenue. The relationship was explained through the extra recruiting budgets, better facilities, and increased athletic and academic support for the athlete once they arrive on campus. Year in and year out, the top programs in the country tend to land some of the best recruiting classes. Caro and Benton (2012) quantified this relationship through regression and reported that recruiting classes can explain $63 \%$ to $80 \%$ of the variance in winning percentages when other factors are controlled for.

Recruiting is also a product of style of play. There is a perception that is driven by the major recruiting pundits that players will often select schools based on the offensive or defensive philosophies of the head coach. Players tend to consider how they will fit within the specific scheme and game plans, as well as their immediate 
contribution to the roster. Equally, there is evidence to suggest that players will select a school based upon the conference's reputation, level of competition, and style of play. Traditionally schools in the Pac-12 and Big 12 run a spread offense with a heavier emphasis on the passing game. The Pac-12 and the Big 12 have established a reputation for high-scoring offenses and yielding top-rated National Football League (NFL) prospects at the quarterback, wide receiver, and other offensive skill positions. This is not to discount the defensive prospects produced by Southern California, Oklahoma and Texas, or the offensive approach of Stanford; however, the reputation of teams from these conferences is one of a high-octane, finesse style offenses. To some degree, many teams from these conferences believe that they can outscore you to win a game. Thus, it can be expected that teams in these conferences may have a recruiting advantage with respect to the offensive skill positions. A national recruit may see the opportunity to play at Oregon or Oklahoma State and know that he will have an opportunity to play in an offense that inflates their statistics and provides a greater opportunity to play in the NFL.

Conversely, the Big Ten and the SEC have established a "four yards and a cloud of dust" reputation. These conferences are known for physical line play, more traditional offensive formations, a heavy emphasis on running the football, and stout defenses. While some teams in these conferences have adopted a spread offense, many rely on traditional offensive sets with a heavier emphasis on the running game. Offensively, the SEC and Big Ten are known to produce top running back and offensive line prospects to the NFL. The SEC is largely regarded as the best defensive conference, largely on the reputation of Alabama and Louisiana State. Still, the reputation of playing defense in the SEC can be enough to attract a defensive prospect to one of the other member schools. These are examples of anecdotal evidence and are not supported by research or the literature.

While the existing literature has established connections between winning and revenue (Caro \& Benton, 2012; Brooker \& Klastorin, 1981; Baade \& Sundberg, 1996; Rhoads \& Gerking, 2000; McCormick \& Tinsley, 1990), and separately, winning and recruiting (Caro, 2012), examining the statistical attributes of conferences and successful teams has been largely ignored. First, this study presents conference statistics to help support or quell some of the anecdotal evidence offered through the media. Included in this case are trends in the sport with respect to the three major facets of the game: offense, defense, and the kicking game. This case will present the relationship between statistics and winning percentages, and the relationship between on-the-field statistics and successful programs by exploring the attributes of successful (defined separately as bowl-eligibility) teams.

\section{METHODS}

This study combined statistics on nearly 80 game-play categories for teams from the AQ BCS conferences (Big East, Big 10, Big 12, SEC, Pac-10, and the ACC) from 2005 to 2011. The year 2005 was selected to control the size of the data set. The list of statistics and their respective definition are found in Appendix A. Statistics were taken from the National Collegiate Athletic Association (NCAA) archive as officially recorded through each game and reported to the NCAA. Each conference was assigned a conference code (SEC, 1; Pac-12, 2; Big 10, 3; Big 12, 4; ACC, 5; Big East 6). Descriptive statistics were formulated for each of the variables in the study. Descriptive statistics and analysis of variance (ANOVA) were used to determine and present any significant differences that existed from year to year. The Games-Howell post-hoc test was used to ascertain in which years these differences existed. Games-Howell was selected as it does not assume equal variances, is appropriate when the assumption of homogeneity of variance is violated, and is recognized as a robust measure when the assumptions of ANOVA are violated (Games \& Howell, 1976; Jaccard, Becker, \& Wood, 1984; Sullivan, Riccio \& Reynolds, 2008). Pearson's product-moment correlation was used to determine the correlation of the continuous measures analyzed in the data set. Teams were then coded "1" for bowl-eligibility and " 0 " for non-eligibility; ANOVA was conducted along these measures. The findings and subsequent questions are presented below.

The statistics (Table 1) selected for this study were taken from the NCAA archive for games played between 2005 and 2011. The reader will note that statistics on efficiencies such as red zone scoring percentage, third down conversions, fourth down conversions, are missing from the file. This is due to the fact that the data was not available at the NCAA website. These could be potential limitations for this study. 
Table 1: Definition of Variables

\begin{tabular}{|c|c|}
\hline Variable & +2 \\
\hline POPatt & Passing offense pass attempts. \\
\hline POPcomp & Passing offense pass completions. \\
\hline POInt & Passing offense interceptions. \\
\hline POPaPct & Passing offense completion percentage. \\
\hline POYds & Total passing yards. \\
\hline POYdsatt & Passing yards per attempt. \\
\hline POTds & Offensive passing touchdowns. \\
\hline POYdsGm & Passing offense yards per game. \\
\hline POIntPct & Passing offense interception percentage (interceptions per attempt). \\
\hline POYdsCmp & Passing offense yards per completion. \\
\hline PDPcomp & Passing defense completions allowed. \\
\hline PDPaPct & Passing defense completion of passes allowed. \\
\hline PDYdsCmp & Passing defense yards per completion allowed. \\
\hline PDInt & Passing defense interceptions. \\
\hline PDIntPct & Passing defense interception percentage (interceptions per attempt). \\
\hline PDYds & Passing defense total yards allowed. \\
\hline PDYdsatt & Passing defense yards per attempt allowed. \\
\hline PDTds & Passing defense touchdowns allowed. \\
\hline PDYdsGm & Passing defense yards per game allowed. \\
\hline ROCarries & Rushing offense carries. \\
\hline RONet & Rushing offense total net yards. \\
\hline ROAvg & Rushing offense yards per carry. \\
\hline ROTds & Rushing offense touchdowns scored. \\
\hline ROYdsGm & Rushing offense yards per game. \\
\hline RDCarries & Rushing defense carries faced. \\
\hline RDNet & Rushing defense net yards allowed. \\
\hline RDAvg & Rushing defense yards per carry allowed. \\
\hline RDTds & Rushing defense touchdowns allowed. \\
\hline RDYdsGm & Rushing defense total rushing yards allowed. \\
\hline SDPoints & Scoring defense total points allowed. \\
\hline SDPtsGm & Scoring defense points per game allowed. \\
\hline SDTds & Scoring defense total touchdowns allowed. \\
\hline SDFG & Scoring defense total field goals allowed. \\
\hline SOYds & Scoring offense total yards. \\
\hline SOTds & Scoring offense total touchdowns. \\
\hline SOPoints & Scoring offense total point scored. \\
\hline SOAvg & Scoring offense average points per game. \\
\hline SOFG & Scoring offense field goals scored. \\
\hline TDAvg & Total defense yards per play allowed. \\
\hline TDPlays & Total defense total plays faced. \\
\hline TDYds & Total defense total yards allowed. \\
\hline TDTds & Total defense total touchdowns allowed. \\
\hline TDYdsGm & Total defense total yards per game allowed. \\
\hline TOPlays & Total offense total plays. \\
\hline TOYds & Total offense total yards. \\
\hline TOAvg & Total offense yards per play. \\
\hline TOTds & Total offense touchdowns scored. \\
\hline TOYdsGm & Total offense yards per game. \\
\hline TMFumGn & Total fumbles gained. \\
\hline TMIntGn & Total interceptions gained. \\
\hline TMGtotal & Total turnovers gained. \\
\hline TMFumls & Total fumbles lost. \\
\hline TMIntls & Total interceptions lost. \\
\hline TMLtotal & Total turnovers lost. \\
\hline
\end{tabular}


(Table 1 continued)

\begin{tabular}{ll}
\hline \multicolumn{1}{c}{ Variable } & Definition \\
\hline TMMargin & Turnover margin \\
\hline TFLPG & Average tackles for loss per game. \\
\hline KRKret & Total kick-off returns. \\
\hline KRYds & Total kick-off return yards. \\
\hline KRTds & Total kick-off return touchdowns scored. \\
\hline KRAvg & Kick-off return average yards per return. \\
\hline NPPunts & Total punts. \\
\hline NPAvg & Punt distance average. \\
\hline NPPuntRet & Punts returned on offensive punts. \\
\hline NPTouchbacks & Return yards on offensive punts. \\
\hline NPNetAvg & Touchbacks on offensive punts. \\
\hline PRRet & Total net punt average. \\
\hline PRYds & Punt returns. \\
\hline PRTds & Punt return total yards. \\
\hline PRAvg & Punt return touchdowns scored. \\
\hline OPassPlayPCT & Punt return average yards per return. \\
\hline ORunPlayPCT & Percentage of offensive passing plays. \\
\hline OPassYdPCT & Percentage of offensive rushing plays. \\
\hline ORunYdPCT & Percentage of passing yards as a function of total yards. \\
\hline
\end{tabular}

\section{RESULTS}

\section{Conference Trends from 2005 to 2011}

ANOVA was conducted to determine if differences existed in the statistics at the conference level for games played from 2005 to 2011; Games-Howell post-hoc tests followed to determine where the significant differences existed. The data is summarized in Table 2 . The data indicate a significant difference in passing offense pass attempts, with the Big $12(\bar{X}=433.26)$ attempting the most passes and the SEC $(\bar{X}=367.90)$ the least. Offensive passing yards per game statistics indicate that the Big $12(\bar{X}=248.07)$ and the Pac $12(\bar{X}=235.73)$ led the conference. Alternatively, the Big $10(\bar{X}=168.84)$ and the SEC $(\bar{X}=157.68)$ led in rushing yards per game. The Big $12(\bar{X}=906.10)$ and the Big $10(\bar{X}=872.28)$ had the most total offensive plays, and the Pac $12(\bar{X}=$ $375.73)$ and the Big $12(\bar{X}=373.39)$ led in total offensive yards per game. The Big $12(\bar{X}=369.98)$ and the Pac 12 $(\bar{X}=358.83)$ led in total offensive points and in scoring points per game at $(\bar{X}=31.06)$ and $(\bar{X}=28.49)$ respectively. Offenses in the SEC and the Big $10(\bar{X}=.56)$ had the highest percentage of run plays while the Pac 12 $(\bar{X}=.52)$ reported the lowest. 
Table 2: ANOVA for Mean Differences for AQ-BCS Football Conferences by Year for 2005 to 2011.

\begin{tabular}{|c|c|c|c|c|c|c|c|c|}
\hline Variable & $\mathbf{F}$ & Sig. & Variable & $\mathbf{F}$ & Sig. & Variable & $\mathbf{F}$ & Sig. \\
\hline POPatt* & 8.254 & .000 & RD Net & .691 & .657 & TM Gtotal & 1.123 & .347 \\
\hline PO Pcomp* & 10.102 & .000 & RD Avg & 1.580 & .151 & TM Fumls* & 2.628 & .016 \\
\hline PO Int & 1.143 & .336 & RD Tds* & 2.692 & .014 & TM Intls & 1.143 & .336 \\
\hline PO PaPct* & 5.338 & .000 & RD YdsGm & .692 & .656 & TM Ltotal & 1.331 & .242 \\
\hline PO Yds* & 6.891 & .000 & SD Points* & 6.696 & .000 & TM Margin & .158 & .987 \\
\hline PO Ydsatt & .485 & .819 & SD PtsGm* & 6.060 & .000 & TFLPG* & 4.227 & .000 \\
\hline PO Tds* & 6.765 & .000 & SD Tds* & 6.877 & .000 & KR Kret & 1.226 & .291 \\
\hline PO YdsGm* & 7.715 & .000 & SD FG* & 3.063 & .006 & KR Yds & 1.483 & .182 \\
\hline PO IntPct* & 4.082 & .001 & SO Yds* & 6.910 & .000 & KR Tds & 1.165 & .324 \\
\hline PO YdsCmp & 1.445 & .196 & SO Tds* & 6.040 & .000 & KR Avg & 2.021 & .062 \\
\hline PD Pcomp* & 15.771 & .000 & SO Points* & 5.105 & .000 & NP Punts* & 2.819 & .011 \\
\hline PD PaPct* & 7.387 & .000 & SO Avg* & 6.292 & .000 & NP Avg* & 2.829 & .010 \\
\hline PD YdsCmp & 1.091 & .367 & SO FG & .934 & .470 & NP PuntRet & 1.730 & .112 \\
\hline PD Int & .886 & .505 & TD Avg* & 4.100 & .001 & NP RetYds & 1.206 & .302 \\
\hline PD IntPct* & 3.684 & .001 & TD Plays* & 6.604 & .000 & NP Touchbacks & .521 & .792 \\
\hline PD Yds* & 14.441 & .000 & TD Yds* & 8.428 & .000 & NP NetAvg & 1.133 & .342 \\
\hline PD Ydsatt* & 2.467 & .023 & TD Tds* & 6.877 & .000 & PR Ret & 1.642 & .134 \\
\hline PD Tds* & 6.992 & .000 & TD YdsGm* & 8.146 & .000 & PR Yds & 1.729 & .113 \\
\hline PD YdsGm* & 16.000 & .000 & TO Plays* & 7.670 & .000 & PR Tds & .913 & .485 \\
\hline RO Carries & 1.924 & .075 & TO Yds* & 6.910 & .000 & PR Avg & 1.511 & .173 \\
\hline RO Net* & 2.567 & .019 & TO Avg* & 4.051 & .001 & OPassPlayPct* & 4.605 & .000 \\
\hline RO Avg* & 4.217 & .000 & TO Tds* & 6.040 & .000 & ORunPlayPct* & 5.061 & .000 \\
\hline RO Tds* & 3.216 & .004 & TO YdsGm* & 9.564 & .000 & OPassYdPct* & 3.360 & .003 \\
\hline RO YdsGm* & 3.147 & .005 & TM FumGn* & 3.167 & .005 & ORushYdPct* & 3.682 & .001 \\
\hline RD Carries & .614 & .719 & TM IntGn & .886 & .505 & \multicolumn{3}{|c|}{ *ANOVA significant at the $\alpha=.05$ level } \\
\hline
\end{tabular}

Defenses in the SEC $(\bar{X}=272.48)$, the Big East $(\bar{X}=282.33)$, and the ACC $(\bar{X}=268.221)$ allowed the fewest total points in the season and points per game. The Big East $(\bar{X}=135.66)$, the ACC $(\bar{X}=136.10)$, and the SEC $(\bar{X}=137.42)$ also allowed the fewest rushing yards per game. The conferences with the fewest passing yards allowed were the SEC $(\bar{X}=192.94)$ and the $\operatorname{ACC}(\bar{X}=205.49)$.

Correlations to winning percentages were then computed for each conference individually for the period of the study. Notable positive correlations in the SEC (Table 3) were passing offense completion percentage $(\mathrm{r}=.678)$, passing yards per attempt $(\mathrm{r}=.662)$, rushing offense net yards $(\mathrm{r}=.591)$, and turnover margin $(\mathrm{r}=.545)$. Notable negative correlations in the SEC were rushing yards per game allowed $(r=-.629)$, rushing yards allowed per carry $(r$ $=-.578)$, and passing defense yards per attempt $(\mathrm{r}=-.536)$.

In the Pac-12 (Table 4), total offensive yards $(r=.750)$, turnover margin $(r=.705)$, rushing yards per carry $(\mathrm{r}=.676)$ were positively related to winning percentage. Passing yards per game $(\mathrm{r}=.273)$, despite the Pac-12 total passing yards, were only moderately, positively related to winning percentage. Notable negative relationships to winning percentage were rushing yards per game allowed $(\mathrm{r}=-.712)$, rushing yards per carry allowed $(\mathrm{r}=-.613)$, net rushing yards allowed $(r=-.575)$, and pass yards per attempt allowed $(r=-.568)$. The Big 10 (Table 5) indicated strong positive relationships to total punt return yards $(r=.631)$, the number of punt returns $(r=.620)$, pass defense interception percentage $(\mathrm{r}=.612)$, pass offense yards per attempt $(\mathrm{r}=.597)$, and turnover margin $(\mathrm{r}=.576)$. Those statistics negatively related to winning percentages in the Big 10 were pass defense yards per attempt allowed $(r=-$ $.724)$, rushing yards per game allowed $(r=-.697)$, pass defense completion percentage $(r=-.591)$, and the number of kick-off returns $(\mathrm{r}=-.415)$.

The Big 12 (Table 6) indicated strong positive relationships between total offensive yards $(\mathrm{r}=.695)$, rushing touchdowns $(\mathrm{r}=.618)$, passing yards per attempt $(\mathrm{r}=.612)$, and turnover margin $(\mathrm{r}=.590)$ and a team's winning percentage. Those factors negatively associated with winning percentages were passing yards per attempt allowed $(\mathrm{r}=-.598)$, passing defense completion percentage allowed $(\mathrm{r}=-.507)$, and rushing defense yards per game allowed $(\mathrm{r}=-.490)$. The ACC (Table 7) indicated strong positive relationships on turnover margin $(\mathrm{r}=.584)$, 
turnovers gained $(\mathrm{r}=.551)$, total offensive plays $(\mathrm{r}=.534)$, scoring offense field goals scored $(\mathrm{r}=.501)$, rushing offense carries $(r=.492)$, and passing yards per attempt $(r=.466)$. Those statistics negatively correlated to winning percentage in the ACC were rushing yards allowed per game $(\mathrm{r}=-.656)$, passing yards allowed per attempt $(\mathrm{r}=-$ $.643)$, average rushing yards allowed per carry $(\mathrm{r}=-.543)$, and kick-off return total yards $(\mathrm{r}=-.459)$. In the Big East (Table 8) total offensive yards $(\mathrm{r}=.728)$, total offensive plays $(\mathrm{r}=.555)$, rushing offense net yards $(\mathrm{r}=.555)$, and tackles for a loss $(\mathrm{r}=.506)$ were positively correlated to winning. Rushing yards allowed per game $(\mathrm{r}=-.580)$, rushing yards allowed per carry $(\mathrm{r}=-.514)$, and passing yards per attempt allowed $(\mathrm{r}=-.477)$ were negatively related to winning.

Table 3: Significant Pearson's Correlations for Variables to Winning Percentage for the SEC Conference from 2005-2011.

\begin{tabular}{ll|lc|lc}
\hline \multicolumn{1}{c}{ Variable } & Correlation & \multicolumn{1}{c}{ Variable } & Correlation & \multicolumn{1}{c}{ Variable } & Correlation \\
\hline SO Points & .781 & TM IntGn & .480 & TM Ltotal & -.332 \\
\hline SO Tds & .749 & SO FG & .456 & KR Kret & -.345 \\
\hline TO Tds & .749 & PO Yds & .450 & PO Int & -.359 \\
\hline SO Avg & .744 & PO YdsCmp & .444 & TM Intls & -.359 \\
\hline SO Yds & .721 & TM Gtotal & .437 & PD YdsCmp & -.360 \\
\hline TO Yds & .721 & NP NetAvg & .320 & PO IntPct & -.376 \\
\hline PO PaPct & .678 & PO Pcomp & .317 & PD PaPct & -.431 \\
\hline RO Tds & .673 & ORunPlayPct & .308 & NP RetYds & -.448 \\
\hline PO Ydsatt & .662 & PO YdsGm & .301 & NP Punts & -.464 \\
\hline TO Avg & .657 & PD Pcomp & .280 & NP PuntRet & -.477 \\
\hline TO YdsGm & .615 & PD IntPct & .278 & RD Net & -.487 \\
\hline RO Net & .591 & TD Plays & .273 & SD Tds & -.499 \\
\hline RO Carries & .556 & PR Tds & .265 & TD Tds & -.499 \\
\hline TO Plays & .553 & TFLPG & .244 & RD Tds & -.503 \\
\hline TM Margin & .545 & PR Avg & .238 & SD Points & -.522 \\
\hline RO Avg & .539 & RD Carries & -.221 & PD Ydsatt & -.536 \\
\hline PO Tds & .527 & PD YdsGm & -.236 & TD YdsGm & -.568 \\
\hline RO YdsGm & .490 & PD Tds & -.241 & RD Avg & -.578 \\
\hline PR Yds & .487 & KR Yds & -.261 & TD Avg & -.579 \\
\hline PR Ret & .480 & TD Yds & -.284 & RD YdsGm & -.629 \\
\hline PD Int & .480 & OPassPlayPct & -.308 & SD PtsGm & -.664 \\
\hline$* *$ Pearson's Correlation significant at the $\alpha=0.05$ level. & & &
\end{tabular}


Table 4: Significant Pearson's Correlations for Variables to Winning Percentage for the Pac-12 Conference from 2005-2011.

\begin{tabular}{ll|ll|ll}
\hline \multicolumn{1}{c}{ Variable } & Correlation & \multicolumn{1}{c}{ Variable } & Correlation & \multicolumn{1}{c}{ Variable } & Correlation \\
\hline SO Points & .845 & PO PaPct & .538 & TM Ltotal & -.382 \\
\hline SO Avg & .823 & TFLPG & .406 & PO Int & -.392 \\
\hline SO Tds & .808 & PO Yds & .405 & TM Intls & -.392 \\
\hline TO Tds & .808 & ORushYdPct & .401 & NP RetYds & -.392 \\
\hline SO Yds & .750 & PR Avg & .383 & TD Yds & -.401 \\
\hline TO Yds & .750 & PD IntPct & .369 & OPassYdPct & -.401 \\
\hline TO Avg & .710 & PR Tds & .358 & PO IntPct & -.456 \\
\hline TM Margin & .705 & TM FumGn & .352 & NP PuntRet & -.458 \\
\hline TO YdsGm & .682 & SO FG & .314 & NP Punts & -.477 \\
\hline RO Avg & .676 & PO Pcomp & .308 & PD PaPct & -.529 \\
\hline RO Net & .673 & KR Avg & .302 & RD Tds & -.533 \\
\hline RO YdsGm & .652 & TD Plays & .294 & TD YdsGm & -.562 \\
\hline RO Tds & .644 & ORunPlayPct & .275 & PD Ydsatt & -.568 \\
\hline PO Tds & .640 & PO YdsGm & .273 & RD Net & -.575 \\
\hline TO Plays & .614 & PD Pcomp & .268 & SD Tds & -.604 \\
\hline TM Gtotal & .599 & KR Tds & .255 & TD Tds & -.604 \\
\hline PR Yds & .583 & RD Carries & -.239 & RD Avg & -.613 \\
\hline RO Carries & .578 & NP Avg & -.267 & SD Points & -.623 \\
\hline PR Ret & .569 & OPassPlayPct & -.275 & TD Avg & -.626 \\
\hline PO Ydsatt & .559 & PD YdsCmp & -.318 & RD YdsGm & -.655 \\
\hline PD Int & .545 & PD Tds & -.353 & SD PtsGm & **Pearson's Correlation \\
\hline TM IntGn & .545 & KR Kret & -.381 & the $\alpha=0.05$ level. & \\
\hline
\end{tabular}

Table 5: Significant Pearson's Correlations for Variables to Winning Percentage for the Big Ten Conference from 2005-2011.

\begin{tabular}{ll|lc|lc}
\multicolumn{1}{c}{ Variable } & Correlation & \multicolumn{1}{c}{ Variable } & Correlation & \multicolumn{1}{c}{ Variable } & Correlation \\
\hline SO Points & .687 & PO Tds & .379 & RD Carries & -.392 \\
\hline PR Yds & .631 & ORunPlayPct & .375 & KR Yds & -.415 \\
\hline SO Avg & .623 & PO PaPct & .356 & KR Kret & -.465 \\
\hline PR Ret & .620 & PR Avg & .335 & PD YdsGm & -.466 \\
\hline SO Tds & .620 & RO YdsGm & .329 & PD Tds & -.548 \\
\hline TO Tds & .620 & TO Plays & .324 & TD Yds & -.563 \\
\hline PD Int & .612 & TO YdsGm & .312 & PD YdsCmp & -.573 \\
\hline TM IntGn & .612 & PR Tds & .298 & PD PaPct & -.591 \\
\hline PO Ydsatt & .597 & NP NetAvg & .293 & RD Avg & -.607 \\
\hline TM Margin & .576 & NP Avg & .292 & RD Net & -.611 \\
\hline PD IntPct & .525 & TFLPG & .287 & RD Tds & -.651 \\
\hline SO Yds & .514 & RO Avg & .262 & RD YdsGm & -.697 \\
\hline TO Yds & .514 & PO IntPct & -.241 & TD Avg & -.702 \\
\hline TM Gtotal & .513 & PD Yds & -.276 & TD YdsGm & -.704 \\
\hline RO Tds & .506 & TM Ltotal & -.288 & PD Ydsatt & -.724 \\
\hline RO Carries & .495 & SD FG & -.311 & SD Tds & -.757 \\
\hline TO Avg & .471 & PO Int & -.333 & TD Tds & -.757 \\
\hline PO YdsCmp & .450 & TM Intls & -.333 & SD Points & -.780 \\
\hline SO FG & .448 & NP Punts & -.362 & SD PtsGm & -.827 \\
\hline RO Net & .437 & OPassPlayPct & -.375 & **Pearson's Correlation & significant \\
\hline
\end{tabular}


Table 6: Significant Pearson's Correlations for Variables to Winning Percentage for the Big 12 Conference from $2005-2011$.

\begin{tabular}{|c|c|c|c|c|c|}
\hline Variable & Correlation & Variable & Correlation & Variable & Correlation \\
\hline SO Points & .788 & TFLPG & .404 & TM Intls & -.272 \\
\hline SO Tds & .757 & PO Yds & .398 & NP Punts & -.290 \\
\hline TO Tds & .757 & TD Plays & .389 & PD Tds & -.342 \\
\hline SO Avg & .753 & RO Carries & .373 & PO IntPct & -.355 \\
\hline SO Yds & .695 & PR Ret & .356 & KR Kret & -.356 \\
\hline TO Yds & .695 & SO FG & .355 & PD YdsCmp & -.363 \\
\hline TO Avg & .630 & PO YdsCmp & .316 & NP PuntRet & -.374 \\
\hline RO Tds & .618 & PR Yds & .316 & NP RetYds & -.378 \\
\hline TO YdsGm & .614 & KR Avg & .298 & RD Net & -.379 \\
\hline PO Ydsatt & .612 & PO YdsGm & .298 & RD Tds & -.457 \\
\hline PD Int & .590 & PO Pcomp & .292 & RD Avg & -.460 \\
\hline TM IntGn & .590 & PR Tds & .250 & RD YdsGm & -.490 \\
\hline PO PaPct & .583 & PR Avg & .250 & TD YdsGm & -.493 \\
\hline TM Gtotal & .583 & TM FumGn & .231 & PD PaPct & -.507 \\
\hline TO Plays & .548 & PD Pcomp & .230 & SD Tds & -.521 \\
\hline TM Margin & .547 & PD YdsGm & -.213 & TD Tds & -.521 \\
\hline PO Tds & .497 & KR Yds & -.217 & SD Points & -.539 \\
\hline RO Net & .444 & TM Ltotal & -.232 & TD Avg & -.584 \\
\hline PD IntPct & .444 & TD Yds & -.250 & PD Ydsatt & -.598 \\
\hline RO Avg & .427 & PO Int & -.272 & SD PtsGm & -.651 \\
\hline RO YdsGm & .420 & \multicolumn{4}{|c|}{ **Pearson's Correlation significant at the $\alpha=0.05$ level. } \\
\hline
\end{tabular}

Table 7: Significant Pearson's Correlations for Variables to Winning Percentage for the ACC Conference from 2005-2011

\begin{tabular}{ll|ll|ll}
\hline \multicolumn{1}{c}{ Variable } & Correlation & \multicolumn{1}{c|}{ Variable } & Correlation & \multicolumn{1}{c}{ Variable } & Correlation \\
\hline SO Points & .708 & RO Avg & .378 & OPassPlayPct & -.282 \\
\hline SO Tds & .649 & PD IntPct & .376 & PD Tds & -.295 \\
\hline TO Tds & .649 & NP Touchbacks & .365 & PD YdsGm & -.303 \\
\hline SO Avg & .639 & PR Tds & .364 & TD Yds & -.343 \\
\hline SO Yds & .603 & RO YdsGm & .352 & PD PaPct & -.383 \\
\hline TO Yds & .603 & TD Plays & .351 & PD YdsCmp & -.431 \\
\hline TM Margin & .584 & PR Ret & .326 & KR Yds & -.459 \\
\hline TM Gtotal & .551 & PO Tds & .319 & KR Kret & -.484 \\
\hline TO Plays & .534 & PO YdsCmp & .302 & RD Net & -.509 \\
\hline PD Int & .510 & TFLPG & .289 & RD Avg & -.543 \\
\hline TM IntGn & .510 & ORunPlayPct & .282 & TD Avg & -.603 \\
\hline SO FG & .501 & PD Pcomp & .281 & SD Tds & -.632 \\
\hline RO Tds & .494 & TM FumGn & .248 & TD Tds & -.632 \\
\hline RO Carries & .492 & ORushYdPct & .236 & TD YdsGm & -.639 \\
\hline TO Avg & .474 & PO Yds & .232 & PD Ydsatt & -.643 \\
\hline PO Ydsatt & .466 & NP Avg & .223 & SD Points & -.654 \\
\hline RO Net & .436 & OPassYdet & -.236 & RD YdsGm & -.656 \\
\hline PR Yds & .428 & PO Int & -.253 & RD Tds & -.664 \\
\hline TO YdsGm & .426 & TM Intls & -.253 & SD PtsGm & -.775 \\
\hline PR Avg & .404 & PO IntPct & -.263 & **Pearson's Correlation significant at \\
\hline
\end{tabular}


Table 8: Significant Pearson's Correlations for Variables to Winning Percentage for the Big East Conference from 2005-2011.

\begin{tabular}{ll|lc|lc}
\hline \multicolumn{1}{c}{ Variable } & Correlation & \multicolumn{1}{c}{ Variable } & Correlation & \multicolumn{1}{c}{ Variable } & Correlation \\
\hline BE & PC & PD Int & .454 & PD Tds & -.328 \\
\hline SO Points & .830 & TM IntGn & .454 & NP PuntRet & -.346 \\
\hline SO Tds & .789 & TM Gtotal & .448 & KR Kret & -.372 \\
\hline TO Tds & .789 & SO FG & .441 & PD PaPct & -.392 \\
\hline SO Avg & .752 & PO PaPct & .431 & RD Net & -.438 \\
\hline SO Yds & .728 & RO YdsGm & .426 & PD Ydsatt & -.477 \\
\hline TO Yds & .728 & TD Plays & .425 & RD Avg & -.514 \\
\hline RO Tds & .672 & RO Carries & .424 & NP Punts & -.534 \\
\hline TO Avg & .648 & PO Tds & .418 & TD YdsGm & -.542 \\
\hline TO YdsGm & .578 & TM Margin & .410 & RD Tds & -.553 \\
\hline TO Plays & .555 & PO Pcomp & .402 & RD YdsGm & -.580 \\
\hline PO Ydsatt & .555 & PO YdsCmp & .399 & TD Avg & -.586 \\
\hline RO Net & .555 & PO YdsGm & .380 & SD Points & -.616 \\
\hline PO Yds & .522 & PR Ret & .324 & SD Tds & -.634 \\
\hline RO Avg & .509 & POPaa & .302 & TD Tds & -.634 \\
\hline TFLPG & .506 & PR Yds & .292 & SD PtsGm & -.731 \\
\hline$* *$ Pearson's Correlation significant at the $\alpha=0.05$ level. & & &
\end{tabular}

\section{Differences among Teams}

Teams were then separated into two cohorts: bowl-eligible teams (defined as six wins or more) and non-bowl eligible teams. ANOVA was conducted to determine if differences existed in the statistics for teams in these two cohorts. ANOVA results (Table 9) indicate that these cohorts were different on every statistic except: offensive passing attempts, rushing defense total carries faced, scoring defense field goals allowed, fumbles lost, kick-off return touchdowns allowed, net punting average, and net punting touchbacks. Passing yards per game for bowl teams $(\bar{X}=228.35)$ was higher than for non-bowl teams $(\bar{X}=210.58)$, as was rushing yards per game $(\bar{X}=166.39$ v. 129.92), total yards per game $(\bar{X}=389.77$ v. 338.74$)$, points per game $(\bar{X}=30.18$ v. 22.18$)$, and total plays $(\bar{X}=$ 891.34 v. 801.21$)$. Bowl teams allowed fewer total yardage $(\bar{X}=338.74$ v. 389.77$)$, fewer points per game $(\bar{X}=$ 21.48 v. 29.16), and allowed fewer rushing yards per game $(\bar{X}=128.45$ v. 165.97). Bowl teams also indicated a positive turnover margin $(\bar{X}=.26$ v. -.34$)$, gained more turnovers $(\bar{X}=25.29$ v. 19.52), and lost fewer turnovers over the course of the season $(\bar{X}=21.90$ v. 23.60$)$.

Statistics most strongly related to winning percentage for bowl teams (Table 9) were passing yards per attempt $(r=.435)$, rushing offensive touchdowns $(r=.435)$, and scoring offense points $(r=.610)$. Scoring defense points allowed per game $(\mathrm{r}=-.504)$, rushing yards per game allowed $(\mathrm{r}=-.436)$, and passing yards allowed per attempt $(r=-.427)$ were most notably negatively correlated to winning percentage. 
Table 9: Significant Pearson's Correlations for Variables to

Winning Percentage for AQ-BCS Conference Bowl-Eligible Teams from 2005-2011.

\begin{tabular}{|c|c|c|c|c|c|}
\hline Variable & Correlation & Variable & Correlation & Variable & Correlation \\
\hline SO Points & .610 & RO YdsGm & .247 & NP PuntRet & -.241 \\
\hline SO Avg & .584 & PD IntPct & .239 & PO IntPct & -.253 \\
\hline SO Tds & .575 & TO Plays & .223 & PO Int & -.260 \\
\hline TO Tds & .575 & TFLPG & .208 & TM Intls & -.260 \\
\hline PO Ydsatt & .435 & PO Yds & .205 & PD Tds & -.278 \\
\hline RO Tds & .435 & RO Carries & .199 & PD YdsCmp & -.289 \\
\hline TO Avg & .433 & PR Avg & .199 & PD PaPct & -.313 \\
\hline SO Yds & .432 & PO YdsCmp & .174 & TD Yds & -.313 \\
\hline TO Yds & .432 & PO YdsGm & .158 & NP Punts & -.315 \\
\hline TM Margin & .378 & SO FG & .147 & KR Kret & -.335 \\
\hline TO YdsGm & .371 & KR Avg & .146 & RD Net & -.382 \\
\hline PO PaPct & .368 & PO Pcomp & .117 & RD Tds & -.396 \\
\hline PD Int & .345 & ORunPlayPct & .095 & RD Avg & -.399 \\
\hline TM IntGn & .345 & OPassPlayPct & -.099 & TD YdsGm & -.409 \\
\hline PR Yds & .338 & PD Yds & -.103 & SD Tds & -.423 \\
\hline PO Tds & .321 & NP RetYds & -.152 & TD Tds & -.423 \\
\hline PR Ret & .307 & SD FG & -.152 & PD Ydsatt & -.427 \\
\hline RO Avg & .300 & PD YdsGm & -.189 & RD YdsGm & -.436 \\
\hline TM Gtotal & .291 & RD Carries & -.224 & TD Avg & -.449 \\
\hline RO Net & .277 & KR Yds & -.238 & SD Points & -.450 \\
\hline PR Tds & .274 & TM Ltotal & -.238 & SD PtsGm & -.504 \\
\hline
\end{tabular}

The sample was then separated into two new cohorts: those teams that won ten or more games and those that did not. ANOVA was conducted to determine if differences existed in the statistics for these two cohorts. ANOVA results (Table 10) indicate that these cohorts were different on every statistic except: offensive passing attempts, pass defense yards allowed, fumbles gained, fumbles lost, kick-off return touchdowns, and net punting touchbacks. Passing yards per game for ten-win teams $(\bar{X}=236.85)$ was higher than for non-ten win teams $(\bar{X}=$ $219.00)$, as was rushing yards per game $(\bar{X}=184.59$ v. 146.99$)$, total yards per game $(\bar{X}=421.44$ v. 366.40$)$, points per game $(\bar{X}=34.69$ v. 25.78$)$, and total plays $(\bar{X}=910.18$ v. 850.97$)$. Ten-win teams allowed less total yardage $(\bar{X}=319.34$ v. 364.12$)$, fewer points per game $(\bar{X}=18.96$ v. 25.18$)$, and fewer rushing yards per game $(\bar{X}=115.23$ v. 146.81) than the non-ten win cohort. Ten-win teams also indicated a positive turnover margin $(\bar{X}=.58 \mathrm{v} .-.07)$, gained more turnovers $(\bar{X}=27.40$ v. 22.44$)$, and lost fewer turnovers over the course of the season $(\bar{X}=19.66 \mathrm{v}$. 23.21). Correlation statistics (Table 11) were calculated for the ten-win cohort. The variables most strongly related to winning percentages for this cohort were scoring offense average points per game $(r=.379)$, rushing offense touchdowns $(\mathrm{r}=.306)$, and punt returns $(\mathrm{r}=.299)$. Those negatively associated with winning percentage were scoring defense points allowed $(\mathrm{r}=-.430)$, kick-off kick returns $(\mathrm{r}=-.422)$, and pass defense yards per attempt allowed $(\mathrm{r}=-.410)$. 
Table 10: ANOVA Comparing Ten-Win Teams to non-Ten Win Teams from AQ-BCS Conferences for 2005 to 2011.

\begin{tabular}{|c|c|c|c|c|c|c|c|c|}
\hline Variable & $\mathbf{F}$ & Sig. & Variable & $\mathbf{F}$ & Sig. & Variable & $\mathbf{F}$ & Sig. \\
\hline POPatt & .152 & .697 & RD Net* & 42.846 & .000 & TM Gtotal* & 66.813 & .000 \\
\hline PO Pcomp* & 6.040 & .014 & RD Avg* & 50.301 & .000 & TM Fumls & 3.509 & .062 \\
\hline PO Int* & 46.019 & .000 & RD Tds* & 50.564 & .000 & TM Intls* & 46.019 & .000 \\
\hline PO PaPct* & 66.164 & .000 & RD YdsGm* & 70.307 & .000 & TM Ltotal* & 40.843 & .000 \\
\hline PO Yds* & 27.467 & .000 & SD Points* & 69.478 & .000 & TM Margin* & 114.460 & .000 \\
\hline PO Ydsatt* & 123.416 & .000 & SD PtsGm* & 101.826 & .000 & TFLPG* & 8.535 & .004 \\
\hline PO Tds* & 70.839 & .000 & SD Tds* & 57.108 & .000 & KR Kret* & 33.335 & .000 \\
\hline PO YdsGm* & 9.003 & .003 & SD FG* & 13.391 & .000 & KR Yds* & 15.861 & .000 \\
\hline PO IntPct* & 40.390 & .000 & SO Yds* & 132.450 & .000 & KR Tds & 1.889 & .170 \\
\hline PO YdsCmp* & 33.327 & .000 & SO Tds* & 212.050 & .000 & KR Avg* & 6.785 & .009 \\
\hline PD Pcomp* & 8.885 & .003 & SO Points* & 234.127 & .000 & NP Punts* & 46.227 & .000 \\
\hline PD PaPct* & 33.711 & .000 & SO Avg* & 186.020 & .000 & NP Avg* & 4.108 & .043 \\
\hline PD YdsCmp* & 29.153 & .000 & SO FG* & 25.886 & .000 & NP PuntRet* & 29.817 & .000 \\
\hline PD Int* & 84.857 & .000 & TD Avg* & 62.812 & .000 & NP RetYds* & 14.930 & .000 \\
\hline PD IntPct* & 41.687 & .000 & TD Plays* & 11.712 & .001 & NP Touchbacks & .000 & .985 \\
\hline PD Yds & .001 & .972 & TD Yds* & 18.415 & .000 & NP NetAvg* & 4.588 & .033 \\
\hline PD Ydsatt* & 66.666 & .000 & TD Tds* & 57.108 & .000 & PR Ret* & 28.771 & .000 \\
\hline PD Tds* & 17.427 & .000 & TD YdsGm* & 62.065 & .000 & PR Yds* & 38.115 & .000 \\
\hline PD YdsGm* & 11.797 & .001 & TO Plays* & 50.259 & .000 & PR Tds* & 30.091 & .000 \\
\hline RO Carries* & 62.762 & .000 & TO Yds* & 132.450 & .000 & PR Avg* & 19.360 & .000 \\
\hline RO Net* & 85.849 & .000 & TO Avg* & 118.365 & .000 & OPassPlayPct* & 19.066 & .000 \\
\hline RO Avg* & 68.781 & .000 & TO Tds* & 212.050 & .000 & ORunPlayPct* & 18.082 & .000 \\
\hline RO Tds* & 136.005 & .000 & TO YdsGm* & 76.318 & .000 & OPassYdPct* & 12.188 & .001 \\
\hline RO YdsGm* & 62.182 & .000 & TM FumGn & 3.052 & .081 & ORushYdPct* & 10.955 & .001 \\
\hline RD Carries* & 10.879 & .001 & TM IntGn* & 84.857 & .000 & \multicolumn{3}{|c|}{ *ANOVA significant at the $\alpha=.05$ level. } \\
\hline
\end{tabular}

Table 11: Significant Pearson's Correlations for Variables to

Winning Percentage for Ten-Win AQ-BCS Conference Teams from 2005-2011.

\begin{tabular}{lc|lc|lc}
\hline \multicolumn{1}{c}{ Variable } & Correlation & \multicolumn{1}{c}{ Variable } & Correlation & \multicolumn{1}{c}{ Variable } & Correlation \\
\hline SO Avg & .379 & RO Net & .162 & PD Yds & -.312 \\
\hline SO Points & .311 & PD PaPct & -.183 & RD Tds & -.324 \\
\hline SO Tds & .307 & NP RetYds & -.206 & RD Net & -.337 \\
\hline TO Tds & .307 & PO IntPct & -.209 & TD YdsGm & -.340 \\
\hline RO Tds & .306 & SD FG & -.210 & RD Carries & -.345 \\
\hline PR Ret & .299 & NP PuntRet & -.223 & PD Tds & -.360 \\
\hline PO PaPct & .287 & PO Int & -.258 & TD Avg & -.381 \\
\hline TM Margin & .287 & TM Intls & -.258 & TD Yds & -.388 \\
\hline PR Yds & .269 & RD Avg & -.260 & SD PtsGm & -.399 \\
\hline TO Avg & .261 & PD YdsGm & -.261 & SD Tds & -.407 \\
\hline RO YdsGm & .251 & TD Plays & -.268 & TD Tds & -.407 \\
\hline RO Avg & .245 & KR Yds & -.290 & PD YdsCmp & -.407 \\
\hline TO YdsGm & .229 & TM Ltotal & -.296 & PD Ydsatt & -.410 \\
\hline KR Avg & .216 & RD YdsGm & -.299 & KR Kret & -.422 \\
\hline PO Ydsatt & .205 & NP Punts & -.307 & SD Points & -.430 \\
\hline TFLPG & .190 & **Pearson's Correlation significant at the $\alpha=0.05$ level. & \\
\hline
\end{tabular}

\section{DISCUSSION}

\section{Conference Trends from 2005 to 2011}

The results indicate that differences exist among the AQ-BCS conferences with respect to style of play, scoring, and correlations to winning percentages. Many of the passing statistics, such as passing attempts, passing yards, and passing offense touchdowns, were led by the Big 12 and the Pac-12 conferences. These two conferences also led in total scoring and scoring points per game. Furthermore, passing plays accounted for a greater percentage of their total plays and total yards. The Big 12 led in total offensive plays, slightly ahead of the Pac-12. 
The data indicates that the Big 12 led, or was in the top three, in rushing statistics. This could be an indication that the league may be more offensively balanced than perceived. While the Big Ten led in total rushing yards per game and rushing yards per carry, the Big 12 was ahead of the SEC to round out the top three. This is interesting as the Big Ten and the SEC are traditionally regarded as running leagues with power backs and power formations. The SEC and the Big Ten led in percentage of run plays as a function of total plays and in percentage of rushing yards as a function of total yards, indicating again, that rushing offenses may be more prevalent in these conferences.

Total defensive points allowed and defensive points per game, a category of strength of defensive play, was led by the SEC, the Big East, and the ACC. The figures from the ACC and the Big East may be influenced by the fact that the ACC and Big East were the least productive offenses, respectively. The SEC is largely regarded as the best defensive conference in the country, and there is now quantitative statistics from 2005 to 2011 to support those claims. Teams from the SEC allow the fewest passing yards per game, are third in rushing yards allowed per game, allow the fewest total yards per game, and have the lowest turnover margin of any conference.

The ACC and the Big East were largely balanced offenses, with a slight lean towards running the football. These conferences collectively, however, scored the least total points and averaged the fewest points per game. While teams like West Virginia and Cincinnati in the Big East and Florida State, Clemson, and Georgia Tech in the ACC can keep pace nationally in scoring, the conference average was plagued by teams who are not able to score at a pace consistent with the national average.

Correlations to winning percentage help to further illustrate the differences amongst the conferences. The relationship between scoring offense and winning percentage was strongest in the Pac $12(\mathrm{r}=.845)$, the Big East $(\mathrm{r}=$ $.830)$ and the Big $12(\mathrm{r}=.788)$, while the strength of the negative relationship between scoring points allowed and winning percentage was greatest in the Big Ten $(r=-.827)$, the ACC $(r=-.775)$, and the Big East $(r=-.731)$. The Big Ten and the ACC were the only conferences where the magnitude of the relationship between defense points allowed and winning percentage was greater than that of the scoring offense.

The SEC had a surprisingly low relationship between defensive points per game $(\mathrm{r}=-.664)$ and winning. This may be an indication of defensive balance in the league, thus raising the importance of having an explosive offense. The SEC correlations indicate that an efficient passing game, rushing touchdowns, and net rushing yards are positively related to winning, while rushing yards per game allowed, rushing yards per carry allowed, and passing yards per attempt allowed are negatively related to winning percentage.

The factors in the Pac-12 are slightly different than those of the SEC. Aside from a stronger relationship between scoring offense points, turnover margin and rushing yards per game were positively related to winning. The results indicated that the Pac-12 was behind the Big 12 in passing yards per game; however, passing yards per game was only moderately correlated to winning $(r=.273)$. Moreover, despite the potent passing teams in the conference, pass defense yards per game allowed was not significantly related to winning percentages. Instead, rushing yards per game allowed $(r=-.655)$ and average yards per carry allowed $(r=-.613)$ were negatively related to winning, while offensive rushing yards per game $(\mathrm{r}=.652)$ indicated a strong, positive relationship to winning.

In the Big Ten, there were eight categories of defensive statistics that were more strongly related to winning percentage than offensive points scored. This helps to stress the importance of defense in the Big Ten. Defensive rushing statistics such as yards per game allowed, rushing touchdowns allowed, net rushing yards allowed, and rushing yards per carry allowed, all had strong relationships with winning percentages. Coupled with the fact that the Big Ten led all conferences in offensive rushing yards per game and rushing yards per carry, the data indicates that stopping the run is of upmost importance in the conference. Field position and forcing your opponent to punt the ball are also important in the Big Ten.

The importance of offense in the Big 12 was highlighted by this study. Aside from leading in total yards, total yards per game, total points scored, and points per game, these statistics are also strongly related to winning percentage. Total offensive yards $(r=.695)$ measures a stronger relationship to winning percentage than points per game allowed $(r=-.651)$, which points to the importance of offensive production in the conference. Limiting 
interceptions and creating interceptions are also related to winning mainly because of the inflated passing attempts of the conference. Possessing the ball (total plays, $r=.548)$ and turnover margin $(r=.548)$ are also important. For success in the Big 12, it appears as though teams must have the ability to score points. In 2011, Oklahoma State, Baylor, and Oklahoma, who led the conference in scoring offense, all won ten games or more; these teams also led the conference in total yards.

Scoring defense points per game allowed $(r=-.775)$ was the most strongly related variable to winning percentage in the ACC, indicating the importance of defense in the conference. Specifically, the ability to stop the run, limit the number of rushing touchdowns, and limit passing yards per attempt allowed are critical. Defenses that can create turnovers, and offenses that can turnovers, are also strongly linked to winning. The ACC leans slightly to being a running conference, with 55\% of the total plays being rushing plays. Field position and field goal scoring, like the Big Ten, is also important in the ACC.

The Big East, statistically was very similar to the ACC. Scoring points, however, is at a premium in the Big East. This may be the reason that scoring offense was so strongly associated with winning percentage in the conference. In 2011, the league fielded two ten-win teams, both of whom led the conference in scoring, and were in the top three in total offense. These findings do not undermine the importance of defense in the league; however, at 26.38 points per game, the Big East was below the AQ-BCS scoring average of 27.78. This decreased production in scoring can be an indication that teams from the Big East field strong, championship-caliber defenses, thus helping them win out of conference games. The conference records in those out of conference games, however, does not support this as the Big East went 3-12 in 15 games versus other BCS schools in 2010 (NCAA, 2012). Statistically, the conference appeared to be the weakest offensively in the BCS.

\section{Differences among Teams}

The results of the ANOVA indicate that there are significant differences amongst bowl-eligible and nonbowl-eligible teams. The bowl-eligible cohort outperformed the non-bowl eligible cohort in nearly every offensive and defensive statistic. The expectation for bowl-eligible teams to outperform the non-eligible cohort was expected; however, the magnitude of the deviation for these cohorts was significant. The correlations indicate the importance of being able to stop the run, limit the big play ability of their opponent, and increase scoring. Bowl eligible teams outscored their opponents by an average of 30.18 to 21.48; those who were not bowl-eligible were outscored by an average of 22.18 to 29.16 . The ability to stop the run has also been a consistent predictor of success amongst all conferences. There was a marked difference between these two cohorts on this statistic at 128.45 to 165.97.

The results for the ten-win cohort in comparison to the non-ten win team cohort are equally, if not more pronounced. These two cohorts were different on each of the variables except passing attempts, pass defense yards, fumbles gained, fumbles lost, kick-off return touchdowns, and punt touchbacks. Again, as with the bowl eligible cohorts, scoring was a major distinction between these groups. Ten win teams beat their opponents by an average score of 34.69 to 18.96 ; the margin for the non-ten win cohort was a leaner 25.78 to 25.18 . Defensive rush yards allowed was also a significant marker for these teams as the ten-win cohort allowed an average of 115.23, while the non-ten win cohort allowed 146.81 yards per game.

\section{CASE QUESTIONS}

1. The results of this study lead to the conclusion that there have been significant changes in game play from 2005 to 2011. Nationally, the most evident patterns in the data are the increase in offensive production, the rise in scoring, and the reliance of efficiency at the quarterback position. Further, the national statistics indicate the importance of stopping the run and limiting big plays in the passing game. What are the implications of these trends on a recruiting front for teams from the various conferences? How would that impact decisions for coaches as they formulate their respecting recruiting plans?

2. The results of this study indicate that there is statistical evidence to conclude that there exist different styles and pace of play through the different conferences. There are also differences in the variables that correlate to success for each conference. Given these relationships, assess the status of realignment in Division One 
football. What are your expectations on where teams landed in conference play? If there were an opportunity to restructure these conference alignments, which ones would make most sense?

3. It can be concluded that there are differences among the various cohorts at the different levels of success. What are the implications of these differences for coaches and for athletic directors? What impact should this have on future scheduling decisions and the types of teams that coaches are willing to play?

4. Coaches now have statistical evidence to support their preparation for conference games. They also have statistical relationships that can guide their style of play. Further, the results of this study help to signal important positions of emphasis in recruiting, and helps establish performance benchmarks to assist coaches in assessing the current state of their program. Design a series of benchmarks for any one of the conferences included in the study. What are the goals that a specific team in that particular conference should aspire to in order to secure a winning season? Further, what are the impact of these on game attendance and the possibility of generating added revenue?

5. Athletic directors now have a benchmark for building successful programs. At a time when great changes continue to occur off the field, athletic directors can now understand the national football landscape. They are also armed with statistical data to inform decisions on scheduling out-of-conference games and understand the important connection between recruiting, revenue, and winning. What are the changes that athletic directors can undertake to help their programs be more attractive for recruits? There is evidence to support an "arms race" in college football. What are the implications of this "arms race" and how can athletic directors structure their programs to be more successful in the future?

6. As football continues to evolve, and the money continues to grow, it is paramount for athletic directors to use every advantage that is within the rules and guidelines of the NCAA. This study provides another such avenue by illuminating the importance of style of play, the relationship between game-play statistics and success, and the factors that differentiate success at different levels of definition. Still, more research is needed to continue to understand the dynamics of college football. Design an in-depth analysis plan to help athletic directors learn more about their program, its strengths and weaknesses, and their needs moving forward. Then, design a change management plan to implement these changes over a realistic period of time.

\section{AUTHOR INFORMATION}

Dr. Cary Caro is an Assistant Professor of Management at Xavier University of Louisiana. Along with his duties as the Managing Editor of the AJBE, he also serves as the Editor in Chief for the Journal of Sustainability Management. His areas of research concentrate on sports economics, management, administration, and sustainability.

\section{REFERENCES}

Baade, R., \& Sundberg, J. (1996). What Determines Alumni Generosity? Economics of Education Review, 15 (1), 75-81.

BCS Background (2012). Retrieved from Bowl Championship Series: http://www.bcsfootball.org/news/story?id=4809699.

Brooker, G., \& Klastorin, T.D. (1981). To The Victors Go the Spoils? College Athletics and Alumni Giving. Social Science Quarterly, 62 (4), 744-750.

Caro, C., \& Benton, C. (2012). The Great Divide: Examining Football Revenue among FBS Schools. International Journal of Sports Science and Coaching, 7 (2), 139-152.

Caro, C. (2012). College Football Success: The Relationship between Recruiting and Winning. International Journal of Sports Science and Coaching, 7 (1), 139-152.

Dumond, J. M., Lynch, A. K., \& Platania, J. (2008). An Economic Model of the College Football Recruiting Process. Journal of Sports Economics, 9 (1), 67-87.

ESPN: Nebraska Approved by Big Ten (2010). Retrieved 2012 from ESPN: http://sports.espn.go.com/ncaa/news/story?id=5276551.

Fulks, D. L. (2010). 2004-2009 NCAA Revenues and Expenses of Division I Intercollegiate Athletics Programs Report. Indianapolis, IN: National Collegiate Athletic Association. 
Games, P., \& Howell, J. (1976). Pairwise Multiple Comparison Procedures with Unequal N's and/or Variance: A Monte Carlo Study. Journal of Educational and Behavioral Statistics, 1 (2), 113-125.

Groza, M. D. (2010). NCAA Conference Realignment and Football Game Day Attendance. Managerial and Decision Economics, 31, 517-529.

Jaccard, J., Becker, M., \& Wood, G. (1984). Pairwise Multiple Comparison Procedures: A Review. Psychological Bulletin, 96 (3), 589-596.

McCormick, R. E., \& Tinsley, M. (1990). Athletics and Academics: A Model of University Contributions. In B. Goff, \& R. Tollison (eds.), Sportometrics (pp. 193-206). College Stations, TX: Texas A\&M University Press.

NCAA Football Statistics (2012). Retrieved 2012 from NCAA: http://www.ncaa.org/wps/wcm/connect/public/NCAA/Resources/Stats/Football/index.html.

Padilla, A., \& Baumer, D. (1994). Big-time College Sports: Management and Economic Issues. Journal of Sport \& Social Issues, 18 (2), 123-143.

Rhoads, T., \& Gerking, S. (2000). Educational Contributions, Academic Quality, and Athletic Success. Contemporary Economic Policy, 18 (2), 248-258.

SBJ: BCS Payouts Grow Along With Big Shares for Big Six Conferences (2011). Retrieved 2011, from Sporting News: http://aol.sportingnews.com/ncaa-football/story/2011-01-24/sbj-bcs-payouts-grow-along-with-bigshares-for-big-six-conferences.

Sullivan, J., Riccio, C., \& Reynolds, C. (2008). Variations in Students' School- and Teacher-Related Attitudes across Gender, Ethnicity, and Age. Journal of Instructional Psychology, 35 (3), 296-305. 